\title{
Digital Publisher
}

ISSN 2588-0705

Estudio de la estrategia feedback a través de la medición del rendimiento laboral en las PYMES

Study of the feedback strategy through the measurement of labor performance in SMEs

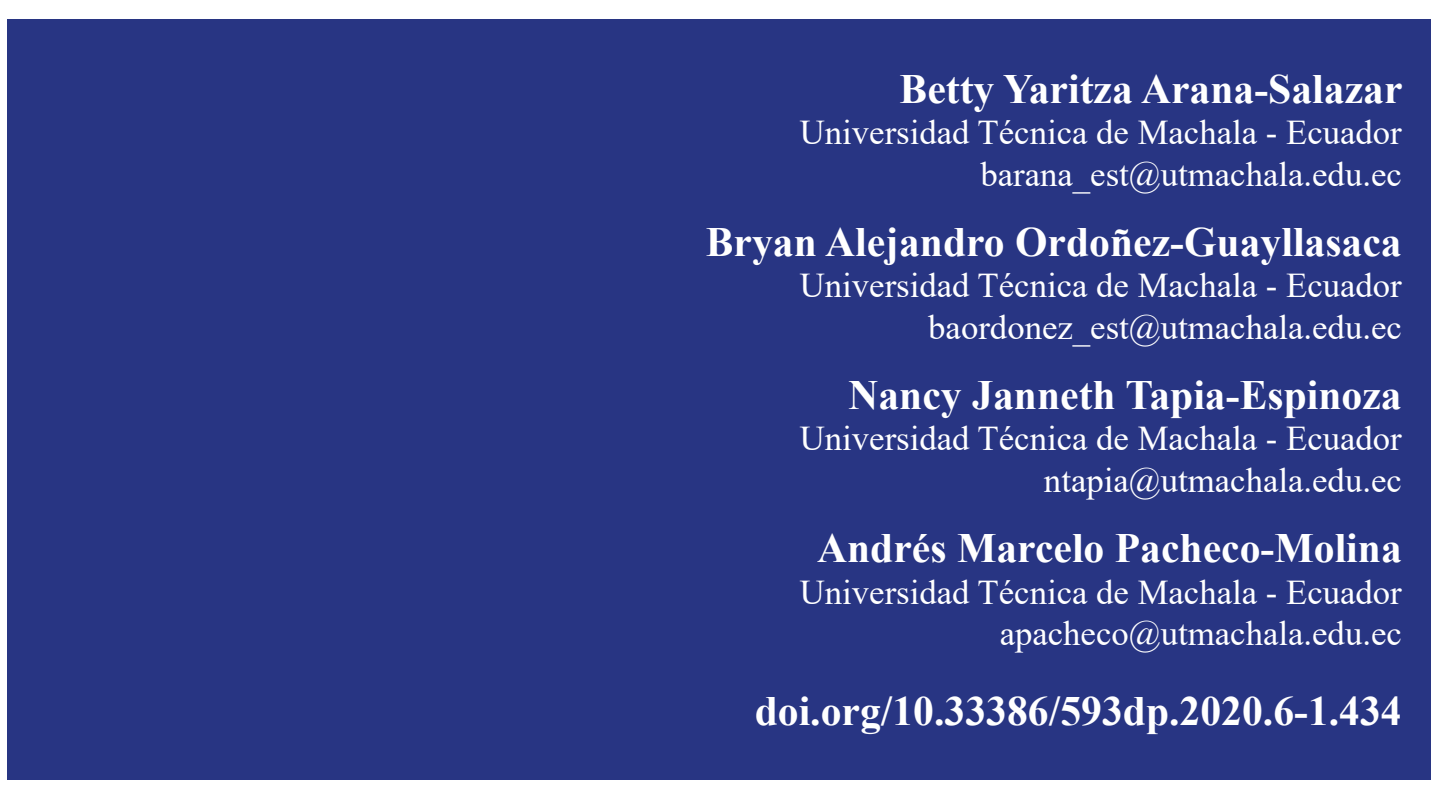

V5-N6-1 (dic) 2020, pp. 360-376 | Recibido: 23 de noviembre de 2020 - Aceptado: 07 de diciembre de 2020 (2 ronda rev.) Edición especial 


\section{RESUMEN}

Las pequeñas y medianas empresas sufren constantes cambios en el mercado donde su objetivo es ser competitiva y brindar un producto o servicio de calidad al usuario, pero para esto deben de tener en cuenta al mayor instrumento dentro de una organización como lo es el recurso humano siendo este aquel que aporta sus habilidades y destrezas en todo proceso o función de tareas, pero si no se mantiene una comunicación eficaz de parte del gerente y los colaboradores con el empleado este no podrá tener un buen rendimiento laboral en función a lo que busca la organización, es por esto que el feedback es un método asertivo que busca con su aplicación reducir errores y aumentar beneficios para los que integran la organización. La presente investigación se desarrolló con el fin de estudiar la estrategia feedback determinando su impacto mediante la medición del rendimiento laboral en las Pymes, implementándose con un enfoque cuantitativo e investigación descriptiva para impulsar el trabajo, se recopiló información a 384 empleados de las Pymes de comercio y servicio en la ciudad de Machala, a través de una encuesta compuesta en dos bloques, el primero sobre rendimiento que consta 9 ítems y el segundo acerca del feedback constituido por 8 ítems, estos datos se analizaron e interpretaron a través de los métodos de correlación en el programa estadístico IBM SPSS lo cual llevó a deducir que el feedback mantiene una relación moderada con el rendimiento de los empleados, aceptando el impacto significativo de esta estrategia dentro de las organizaciones.

Palabras clave: empleado, organización, feedback, rendimiento, comunicación

Cómo citar este artículo:

APA:

Arana, B., Ordoñez, B., Tapia, N., \& Pacheco, A. (2020). Estudio de la estrategia feedback a través de la medición del rendimiento laboral en las PYMES. 593 Digital Publisher CEIT, 5(6-1), 360-376. https://doi. org/10.33386/593dp.2020.6-1.434

Descargar para Mendeley y Zotero

\section{ABSTRACT}

The companies can be small and medium-sized they have constant changes in the market where their objective is to be competitive and provide a quality product or service to the people called user, but for this they must account with the greatest instrument in an organization such as human resources, who contributes with their abilities and skills in any process or task function, the communication is the method more effective to the relationship the manager with their collaborators, if there are not an effective communication between the manager and the collaborators will not have a good job performance based on what the organization require, for this reason, feedback is an assertive method that seeks with its application to reduce errors and increase benefits for who make up the organization. This research was developed in order to study the feedback strategy determining its impact by measuring performance job in Pymes, it is implementing with a quantitative approach and descriptive research to promote work, the information was collected from 384 employees of Pymes of commerce and service in Machala, through a survey with two blocks, the first block consist of 9 items and the second is about feedback, it is made for 8 items, these data were analyzed and interpreted through the correlation methods in the IBM SPSS statistical program, which we have had the deduction that Feedback maintains a moderate relationship with employee performance, accepting the significant impact of this strategy within organizations.

Key words: employee, organization, feedback, performance, communication 


\section{Introducción}

Las Pymes son las empresas que más valor otorgan a un país, teniendo con ello la posibilidad de otorgar más plazos de empleo a una población, caracterizándose por su rapidez en el momento de adaptarse a los cambios que surgen dentro del mercado, aunque cuentan con múltiples ventajas también tienen deficiencias que se rigen por su cultura, como lo refirió Zapata (2004) en ciertos aspectos como lo son el deseo inherente del gerente en crecer de forma rápida su actividad sin medir los recursos que engloban a la organización, así mismo una deficiencia interna en la participación de los empleados con la planeación de actividades y por ende una evaluación inexistente sobre las tareas del recurso humano.

Esas deficiencias dan la apertura a la falta de correspondencia entre gerente y empleado, surgiendo la probabilidad de que la organización no progrese en el cumplimiento de sus metas, destacándose ahí la importancia de realizar una estrategia asertiva interna, para que esa comunicación se fortalezca, siendo la más oportuna la aplicación delfeedback en los procesos y así evidenciar posibles desorientaciones para poder manejar, controlar y corregir las destrezas que destina el recurso humano hacia su puesto de trabajo (Muñoz, Cabrita, y Ribeiro, 2015).

Es por esto que para el desarrollo de la investigación se estudia las Pymes del cantón Machala perteneciente a la provincia de El Oro, así mismo es la que concentra más actividad productiva como lo manifestó el Instituto Nacional de Estadísticas y Censos (INEC) citado en Urdaneta, González, Luciani, y Borgucci (2019) siendo los sectores de comercio y servicio los que ayudaran a la obtención de información relevante al fin de estudiar la estrategia feedback determinando su impacto mediante la medición del rendimiento laboral en las Pymes; lo que implica: desarrollar el marco teórico acerca de la estrategia feedback y el rendimiento laboral mediante la revisión literaria para la orientación lectora de la investigación; recopilar información de las variables mediante la encuesta para la obtención de datos estadísticos; $y$, determinar la relación que existe entre las variables con la ayuda del programa estadístico SPSS para la extracción de resultados.

Esta investigación se rige bajo el enfoque cuantitativo teniendo en cuenta las siguientes hipótesis planteadas H1:El feedback tiene impacto en el rendimiento de los empleados, Ho: El feedback no tiene impacto en el rendimiento de los empleados, para esto se utilizan los instrumentos de los autores Steelman, Levy, y Snell (2004) con feedback environment (FES) y Gabini y Salessi (2016) escala de rendimiento laboral individual, de estas dos guías se analizan los ítems esenciales para la aplicación del feedback a través de la medición del rendimiento con el método estadístico del coeficiente de correlación de Pearson para cuantificar la relación entre las variables.

Es así que el estudio se engloba en la definición del feedback desde las características hasta sus beneficios dentro del entorno empresarial, como en conceptualizar el rendimiento y la forma en que se lo mide dentro del instrumento aplicado a las Pymes de comercio y servicio de la ciudad de Machala, aunque las empresas atraviesan ciertos inconvenientes que impiden receptar la información de forma personal se procederá a obtenerla vía online.

\section{Estrategia Feedback a través de la medición del rendimiento laboral en las Pymes.}

\section{PYMES}

González citado en Ibarra, González, y Demuner (2017) indicó que las pequeñas y medianas empresas son las organizaciones que surgen con mayor ímpetu en todos los países, con un 95\% de los negocios a nivel mundial, siendo así mismo predominante en los mercados nacionales, ese dominio se debe a varios beneficios que se les otorga a este tipo de sociedades, los cuales se las diferencia de su competencia, siendo esta la capacidad de empleados que pueda tener y la posibilidad de adaptarse de forma rápida a los cambios de la economía como de la demanda.

En Latinoamérica estas empresas son la columna 
vertebral de la producción interna de un país, permitiendo un mejor bienestar económico y social para la población, satisfaciendo las diferentes necesidades del ser humano a través de un puesto de trabajo, pero la participación de estas organizaciones en los mercados internacionales es limitada por la deficiencia de información sobre leyes o tratados que se rigen en cada comercio, sin embargo no dejan de ser parte del desarrollo de una cultura existente de personas preparadas con todos los conocimientos adquiridos a través de estudios o experiencia que los hacen ser eficientes para el crecimiento de la organización (García, Galarza, y Altamirano, 2017).

En Ecuador las Pymes se clasifican según la Cámara de Comercio de Quito citado en Brito, Narváez, Erazo, y Torres (2020) en monto de activos, valor bruto en ventas y personal ocupado siendo este último de 10 a 49 si son pequeñas y de 50 a 199 si son medianas (ver Tabla 1). Estas rigen en el país bajo un control por parte de la Superintendencia de Compañías, Valores y Seguros, institución que promueve la transparencia en cada movimiento que realizan las empresas para así promover la confianza en la actividad empresarial del país, prevaleciendo la mejora continua en los procesos que se desarrollan hasta brindar el producto o servicio al usuario.

\section{Tabla 1}

Parámetros de clasificación de las Pymes

\begin{tabular}{|c|c|c|c|c|}
\hline Variables & $\begin{array}{c}\text { Micro } \\
\text { Empresas }\end{array}$ & $\begin{array}{l}\text { Pequeña } \\
\text { empresa }\end{array}$ & $\begin{array}{l}\text { Mediana } \\
\text { Empresa }\end{array}$ & $\begin{array}{l}\text { Grandes } \\
\text { Empresas }\end{array}$ \\
\hline \multicolumn{5}{|l|}{$\begin{array}{l}\text { Personal } \\
\text { Ocupado }\end{array}$} \\
\hline & De 1 a 9 & De $10-49$ & De 50-199 & $\geq 200$ \\
\hline $\begin{array}{c}\text { Valor Bruto } \\
\text { Ventas }\end{array}$ & $\leq 100.000$ & $\begin{array}{l}100.001- \\
1.000 .000\end{array}$ & $\begin{array}{c}1.000 .001- \\
5.000 .000\end{array}$ & $>5.000 .000$ \\
\hline $\begin{array}{l}\text { Monto de } \\
\text { Activos }\end{array}$ & $\begin{array}{l}\text { Hasta } \\
\$ 100.00\end{array}$ & $\begin{array}{c}\text { De } \\
\$ 100.001 \\
\text { hasta } \\
\$ 750.000\end{array}$ & $\begin{array}{c}\text { De } \$ \\
750.001 \\
\text { hasta } \\
\$ 399.999\end{array}$ & $\geq 4.000,000$ \\
\hline
\end{tabular}

Fuente: Tomada de (Brito, Narváez, Erazo, y Torres, 2020)
Estas empresas en el ámbito nacional son un total de 41.643 conforme lo manifestó el INEC citado en Urdaneta et al. (2019) también se indicó que el 8,4\% son de pequeñas empresas y el 1,8\% de medianas empresas, es así que dentro de la provincia de El Oro los cantones con más actividad productiva de los 14 que la componen son Machala 45\%, Pasaje 10\% y Santa Rosa $10 \%$, prevaleciendo los sectores de comercio con un $41 \%$, el de servicios con un $34 \%$ y el restante al área agropecuaria y de otros recursos biológicos.

\section{Estrategia}

La estrategia se ha derivado de varios conceptos a través del tiempo englobándose en diferentes tipos, pero se tiene que resaltar su inicio que fue sujeto por un precedente, como lo fue la lucha de varias naciones que se regían por un conflicto social, es así que el origen según Tzu citado en Bravo, Valenzuela, Ramos, y Tejada (2019) se sostiene en que "el significado etimológico de estrategia proviene de la palabra griega strategos que significa líder de ejército esquema que ha sido implementado por las organizaciones militares para intentar alcanzar sus objetivos" ( $p$. 3). Al ejecutar una estrategia sin importar cuál es la finalidad se mantiene el mismo concepto que es el de alcanzar el cumplimiento de objetivos a través de los medios o recursos necesarios para lograr los mejores resultados.

Un tipo de estrategia es la empresarial que no se aleja del concepto principal, la cual guía a la organización a través de un líder, recursos y planeación, enfocándose en los objetivos como en el mercado meta, que tiene como propuesta colocar todo esfuerzo para lograr la expansión del producto o servicio, manteniendo una relación directa con las actividades que se realizan en cada área, de acorde a las políticas establecidas dentro de la sociedad, sean estas a corto o largo plazo, influyendo también sus cambios de acorde a las tendencias que emergen en el mercado como en la competencia (Mora, Vera, y Melgarejo, 2015).

\section{Feedback}

Según Giraudier (2018) definió al feedback 
como aquel medio de comunicación que se compone del emisor, siendo esta persona la que recoge las reacciones de la otra persona con la que interactúa como lo es el receptor, en esta comunicación bilateral del mismo modo se intercambia información relevante, bajo un desarrollo amplio de habilidades para realizar un comunicado que se ajuste al entorno del destinatario. Al transmitir el mensaje se tiene que percibir la reacción que tiene el observador para asegurar que no se perjudico a nadie ya que toda persona consta de diferentes percepciones y quizás no se entendió por completo el punto de vista de la fuente inicial, siendo este la pauta para tener un feedback entre dos personas en la cual se resalten diferentes criterios. Es por esto que según Zuluaga (2017) esta estrategia debe estar adaptada a tareas especificas del empleado donde se tome en cuenta las circunstancias que lo rodean para tener claro los meritos y errores en las labores realizadas.

\section{Feedback Laboral}

El feedback laboral se centra en ser un suceso del cual el empleado es participe de cómo está siendo el desarrollo de su actividad laboral dentro de la organización, este proceso permite notificar al gerente la eficacia con la que se efectúa las actividades, caso contrario se determinan ciertas acciones correctivas para que se elabore de forma sistemática las diferentes acciones, destacándose de esa manera la responsabilidad en las tareas diarias, teniendo un rol importante el gerente, el cual debe de supervisar los cargos para sincronizar el esfuerzo con la productividad (Caldas, Navarro, y Hidalgo, 2016).

Es por esto que es notable la presencia de este proceso en un entorno empresarial en el que se provee información a diferentes áreas, por lo que siempre se refleja un emisor y receptor, por eso se maneja la percepción de información, desde el punto de vista del gerente o de un encargado, siendo estas las personas que deben de observar el trabajo de cada uno de sus empleados, puesto que es esencial que estos tengan una enseñanza previa para ofrecer un trabajo de calidad, así la institución garantiza un esfuerzo real hacia su grupo de talento humano y no solo una fachada para cumplir con las normas (García S. , 2017).

El encargado al mantener esa cadena de montaje, se limita a llegar a la información existente y se rehúsa al contacto con el talento humano, esta brecha de desacuerdo debe ser solucionada con una comunicación bilateral la cual sea la indicada para transformar esos problemas en una alianza que fortalezca la relación, induciendo a la obtención de buenos resultados, de lo contrario crecerá esa interpretación errónea acerca de los conocimientos de ambas partes ya que el sentido del feedback es dedicar espacios en que se predomine la toma de decisiones en base a fundamentos que parten de ideas conjuntas e interactivas, en que el aprendizaje brinde oportunidades de crecer en el plano profesional, dejando una marca organizacional en base a la trayectoria de la actividad comercial, haciendo la diferencia de la competencia con la autorrealización en cada actividad y por ende la autocorrección en debidas operaciones (Garbanzo, 2016).

Poresosetienequeprevalecerlaretroalimentación y no desaprovechar la oportunidad de poder crear zonas que inciten a una buena comunicación que sea de beneficio para ambas partes, destacándose las fortalezas y debilidades que tiene cada uno para buscar una solución a los fallos y poder desarrollar con mayor ímpetu aquellas destrezas convirtiéndolas en un gran aporte hacia el objetivo de la empresa.

\section{Característica del Feedback}

El feedback se caracteriza por desarrollarse con el fin de dejar secuelas en las personas que lo reciben y ampliar la confianza de ellos en un determinado entorno, por eso en el ámbito empresarial el gerente tiene que estar en contacto desde el primer día con el empleado para que surja esa interacción fluida en toda información que sea relevante para la empresa, como el objetivo que quieren alcanzar en la organización, desarrollándose la apertura para denotar ciertos temas como el proceso, formación, valores $\mathrm{y}$ políticas que se manejan e informarle las 
evaluaciones que se realizan en determinados periodos, de tal manera que el feedback sea descriptivo, simple, centrado y claro sobre las indicaciones que se le otorgaran en todo momento a los receptores (Jiménez, 2015).

\section{Beneficios del Feedback}

La investigación realizada por Casares (2007) determinó que el feedback tiene los siguientes beneficios:

1) La comunicación se puede basar en datos ya existentes lo que supone pocas variaciones en los procedimientos ya empleados, 2) es un proceso sencillo que requiere poca inversión de dinero o de tiempo, 3) tiene validez ya que es un medio natural de control, 4) los resultados se obtienen con rapidez en comparación a otros programas. 5) refuerza otros procesos de la gestión de personal como es la capacitación y desarrollo. (p. 7)

Los beneficios que determinan al feedback no tienen gran complejidad de desarrollo pero si tiene una gran repercusión positiva para la organización, desde el momento de su aplicación, siendo mayor a la que se consigue al no emplearla, puesto que los resultados que se arrojan son más rápidos que cualquier otra evaluación que se realice y es menos costoso, teniendo un efecto en todos los procesos que genere el personal, comprendiendo a todos los niveles o rangos desde la posición de superioridad hasta inferioridad, pero nunca se tiene que desplazar a ningún operario para que el feedback sea realizado de forma general y tenga el resultado que se desea, junto con la disminución de cualquier barrera que esté creciendo dentro de ella.

\section{Tipos de Feedback}

El feedback se desprende en diferentes tipos, a continuación, se abarcarán aquellos que son de realce en el ámbito empresarial, teniendo en la primera clasificación el feedback positivo y negativo que según Amo (2018) los determinaron de la siguiente manera:

Feedback positivo: es reconocer a la otra persona una determinada conducta, lo que hará que su entusiasmo y motivación aumenten. Se puede hacer en público o en privado, si bien en ocasiones hacerlo delante de otras personas ayudará a que dicho comportamiento sea identificado también como modelo a seguir. Feedback negativo: sirve para tratar aspectos que las personas deben corregir, cambiar o mejorar en su comportamiento. También se le llama constructivo, si bien en realidad todo feedback debería tener esta connotación. (p. 57)

El feedback positivo es conmemorar al trabajador por su labor efectiva y por su alto rendimiento o aportación de ideas a la empresa, este suceso resulta demasiado satisfactorio para una persona que se esfuerza día a día para poder cumplir con los estándares de la agrupación, así se expandirá el ejemplo a otros empleados, al ver el reconocimiento que se les procura dar por ese plus que realizan en las actividades. Caso contrario sucede con el feedback negativo en el que se toman en cuenta los errores que realiza el empleado para que no genere un retroceso en los procesos, pero no se llega al extremo de despedirlo sino a brindarle un criterio constructivo para que mejore.

Otracategorización es elfeedback de comprensión determinado por Urcola (2015) como aquel que se comete para comprobar si el mensaje brindado al personal llega según lo planificado en cada área de trabajo. Esta tipificación se centra en el entendimiento que se realiza en el cambio de información de cualquier actividad o evento, teniéndose en cuenta que la otra persona reciba el mensaje de forma clara y concisa para que no existan tergiversaciones al utilizar un lenguaje sencillo acorde al receptor, pero retórico para expresarse de manera conveniente, de modo que el mensaje que se quiera transmitir de una persona a otra sin importar el cargo o rango sea percibido y comprendido.

\section{Rendimiento}

El rendimiento es aquella relación que existe entre lo que se produce dentro de una organización y los medios que se utilizan para la aportación de los objetivos, a través de este término se desarrolla el céteris paribus siendo este que, a un alto grado de bienestar, se genera un mayor rendimiento 
laboral, así las condiciones de la empresa y del empleado serán iguales (Sánchez, Sánchez, Cruz, y Sánchez, 2014). Es así que, para incrementar el rendimiento laboral, hay que tener en cuenta a cada individuo que conforma el grupo colectivo de trabajo mediante la comunicación y el reconocimiento, prevaleciendo una igualdad de los factores que influyen en sus tareas, es decir si al empleado le contemplan sus beneficios este responderá a través de sus esfuerzos, logrando mayor productividad y competitividad.

El factor que va de la mano con el rendimiento es el desempeño laboral, para lograr la productividad que busca una organización se debe de considerar los medios que dispone, estos dos términos se unen en un mismo concepto en la que se realizan las actividades o también como lo definió Koopmans et al. citado en Gabini, y Salessi (2016) aquellas conductas que son primordiales para el cumplimiento de las metas establecidas y se encuentran bajo el manejo de cada uno de los integrantes involucrados en los procesos.

La ejecución de este desarrollo se dirige por la gestión al momento de coordinar todas las responsabilidades así lo indicó Aguinis citado en Gabini (2018) entendiéndose como aquel suceso continuo que se verifica a partir de la identificación de las actividades hasta el desarrollo de las mismas, que permite el equilibrio del rendimiento y la alineación de las estrategias. Esta gestión permite la relación comunicativa con los gerentes y los empleados, ambas partes, instruyendo desde un inicio cuáles son los factores claves que impulsarán al negocio.

\section{Evaluación del Rendimiento}

La evaluación del rendimiento dentro de una organización permite visualizar las distinciones que existen entre el procedimiento estructural instituido y la contribución relativa del sujeto, siendo prioritario supervisar, controlar y evaluar lo que realizan los empleados para poder superar todas las barreras empresariales ya que al presentar un pare en el proceso que se realiza de forma continua significaría una pérdida económica como de confianza hacia la empresa y clientes, por eso es fundamental evaluar de forma periódica el desempeño para así poder aplicar acciones correctivas las cuales resolverán de forma rápida y eficaz cualquier error en el proceso (De Alba, 2015).

La evaluación de este término sigue un modelo que busca reconocer el mérito que tiene un empleado en la organización, comprendiendo esta valoración desde varios puntos de vista en la cual interviene todo el recurso humano que colabora en el proceso sistemático. Esto significa que la evaluación comprende según Gutiérrez citado en Rivero (2019) una apreciación de $360^{\circ}$, llevada a cabo por cada uno de los integrantes del grupo empresarial, sin importar jerarquías sino el aporte hacia cierto objetivo, por lo que se da una interacción de opiniones entre jefes, supervisores, clientes y compañeros, incluyendo la autoevaluación para valorar los conocimientos y destrezas que se contemplan en cada uno, situación que es compleja para aquellos que no tienen motivación o autoconfianza, pero esto permite visualizar la ética y transparencia que tiene cada uno como persona.

Pero para poder evaluar el rendimiento se tiene que regular en primera instancia este constructo, partiendo que al momento de medir se debe de prevalecer los objetivos que tiene la empresa y de ahí partir hacia los factores que permitirán una buena estructura de evaluación con el propósito de abarcar todos los aspectos importantes de los recursos.

\section{Medidas Objetivas}

La objetividad del rendimiento se basa en la medición, pero de forma cuantitativa, aquí se aplica aquello que se determina en hechos y no en palabras, es decir las tareas conseguidas a través de valores numéricos que van de la mano con metas u objetivos establecidos en un lapso de tiempo. Este tipo de medidas se amplifica bajo dirección por objetivos por eso se toma en cuenta los productos vendidos, la adquisición de nuevos clientes y aquellas piezas que han sido fabricadas (Hernández, 2016).

Estas medidas se encuentran dentro de un rango 
delimitado con respuesta precisa y no se ve afectado el empleado por ningún asunto personal, cargo u alteración de resultados, la formulación de las preguntas que se manejan bajo esta medida se concentran en ajustarse a las características de los objetivos, por ende, se analiza cada uno de las actividades que se han desarrollado y se obtiene un resultado concreto del aporte del empleado hacia la organización.

\section{Medidas Subjetivas}

Esta se basa más en las percepciones que se tiene como persona, donde la medición influye más en los factores que ha realizado el recurso humano, pero no lo que han logrado, en otras palabras, se puede decir que la información otorgada depende mucho del interés propio, tanto del evaluado como del evaluador. El encargado de analizar se enfoca en ciertas particularidades que desarrolla el empleado en toda actividad y la perspectiva del individuo al presentarse un problema o un nuevo desafío, caracterizándose por el hecho de que las metas cumplidas no es lo más notable sino el esfuerzo que realizan, de forma más clara alguien que busca solucionar con sus capacidades y destrezas las funciones en su puesto de trabajo, siendo rasgos conscientes al momento en que el evaluador le toca informar acerca de las carencias y méritos que se han realizado en las ocupaciones profesionales (Hernández, 2016).

\section{Metodología}

La presente investigación es descriptiva la misma que es definida según Gabriel (2017) de tal forma que caracteriza al objeto de interés y a sus componentes, además tiene un enfoque cuantitativo sobre el estudio de feedback en las pequeñas y medianas empresas en la cual se busca determinar las siguientes hipótesis: $\mathrm{H} 1$ :El feedback tiene impacto en el rendimiento de los empleados, Ho: El feedback no tiene impacto en el rendimiento de los empleados, estas serán medidas bajo la relación de las diferentes variables, haciendo hincapié en la inferencia que revela ante una población determinada.

\section{Método}

\section{Población}

La población para la investigación es infinita ya que no existen estadísticas actuales sobre las pequeñas y medianas empresas de comercio y servicios en el cantón Machala, los últimos valores no reflejan la realidad que se está viviendo hoy en día, teniendo en cuenta que algunas de ellas pueden ya no laborar por la crisis económica que ha impulsado a los gerentes o dueños de las organizaciones a cerrar los negocios.

\section{Determinación de la Muestra}

Altenerunapoblacióninfinitalamuestrasecalcula según Aguilar (2005) a través de la fórmula:

$$
\mathrm{n}=\frac{\mathrm{Z}^{2} \mathrm{pq}}{\mathrm{d}^{2}}
$$

Donde el nivel de confianza de $95 \%$ se reemplaza en la fórmula con el valor de 1,96 en $\mathrm{Z}$, d como margen de error 5\% (0.05), p: probabilidad que ocurra el suceso esperado, q: probabilidad que el suceso no ocurra, cada uno con un valor de 0,5 ya que al no tener una población determinada se le otorga el $50 \%$.

$$
\mathrm{n}=\frac{1,96^{2}(0,5)(0,5)}{0,05^{2}}=384,16
$$

La fórmula otorgo una muestra a investigar de 384 empleados en total de las pequeñas y medianas empresas de la ciudad de Machala.

\section{Indagación de los instrumentos acorde al tema.}

El tema de estudio se centró en dos variables que son el feedback y el rendimiento, acorde a esto se indago varios artículos con el mismo enfoque, para que sirvan de guía en la investigación, en virtud de esto se encontró dos herramientas que se complementaron para formar un solo instrumento, cuya medida es de forma psicométrica para visualizar la manera en que el feedback influye en el rendimiento laboral de los empleados. 


\section{Feedback Environment (FES)}

Siendo este aquel elemento que "mide una visión mucho más integral del entorno de retroalimentación y es más relevante para las organizaciones de hoy y las responsabilidades de los gerentes del siglo XXI" (Steelman, Levy, y Snell, 2004, p. 166). Esta herramienta se compone por 4 secciones la cual se enfoca en la fuente de credibilidad, calidad de la retroalimentación, comentarios favorables y disponibilidad del servicio, de manera que la valorización de cada ítem se realizó a través de la escala de Likert que varía desde totalmente en desacuerdo hasta totalmente de acuerdo.

\section{Escala de Rendimiento Laboral Individual}

Esta escala tiene dos versiones el primer instrumento acerca del rendimiento, pero en versión inglés que fue construido por los autores Koopmans et al. (2012) basándose en un marco conceptual de cuatro dimensiones: desempeño de tareas, desempeño contextual, desempeño adaptativo y comportamiento de trabajo contraproducente. El segundo es la versión en español del instrumento de Koopmans realizado por los autores Gabini y Salessi (2016) el que se destaca por medir el rendimiento en base de percepciones psicométricas con sus dimensiones de rendimiento en la tarea, rendimiento en el contexto y comportamientos contraproducentes que constituyen la misma idea que tienen los ítems originales.

\section{Instrumentos establecidos.}

Feedback Environment (FES) de Steelman, Levy, y Snell (2004) compuesto por 4 ítems: fuente de credibilidad, calidad de retroalimentación, comentarios favorables $\mathrm{y}$ disponibilidad del servicio.

Escala de Rendimiento Laboral Individual en versión español de los autores Gabini y Salessi (2016) para un mejor entendimiento en la cual se tomaron los siguientes ítems: rendimiento en la tarea, comportamiento contraproducentes y rendimiento en el contexto, estos se destacan por su evaluación de los últimos 3 meses.

\section{Ejecución de encuestas hacia la Muestra.}

El instrumento fue enviado a los empleados de las pequeñas y medianas empresas del cantón Machala, las cuales desarrollan actividades de comercio y de servicios, este proceso fue realizado de forma on-line, por lo que se respetó el distanciamiento social como las normas de bioseguridad de las empresas.

\section{Obtención de Resultados}

Por medio del programa SPSS se aplicó el método de correlación de Pearson para conocer con mayor exactitud la correspondencia de los ítems y para ser más exacta su grado de relación se tomó en cuenta la Tabla 2, que ayudara a entender los valores y con ello llegar a la aceptación o rechazo de las hipótesis.

Tabla 2

Grado de relación según coeficiente de correlación

\begin{tabular}{cc}
\hline RANGO & RELACION \\
\hline-0.91 a -1.00 & Correlación negativa perfecta \\
-0.76 a -0.90 & Correlación negativa muy fuerte \\
-0.51 a -0.75 & Correlación negativa considerable \\
-0.01 a -0.10 & Correlación negativa media \\
0.00 & Correlación negativa débil \\
+0.01 a +0.10 & No existe correlación \\
+0.11 a +0.50 & Correlación positiva débil \\
+0.51 a +0.75 & Correlación positiva media \\
+0.76 a +0.90 & Correlación positiva considerable \\
\hline 0.91 a +1.00 & Correlación positiva muy fuerte \\
\hline
\end{tabular}

Tomado de Hernández y Fernández citado en Mondragón (2014).

\section{Resultados}

Los resultados obtenidos en la investigación, fueron a través de encuestas aplicadas a las pequeñas y medianas empresas de comercio $\mathrm{y}$ 
servicios de la ciudad de Machala, las cuales arrojaron datos que permitieron conocer información necesaria sobre las Hipótesis: H1: El feedback tiene impacto en el rendimiento de los empleados, Ho: El feedback no tiene impacto en el rendimiento de los empleados; esto se determino a través del programa IBM SPSS, que facilitó la recopilación, análisis e interpretación de datos por medio del método estadístico de correlación de Pearson siendo aquello que ayuda a saber si las variables utilizadas tienden a relacionarse entre sí, esta herramienta refirió las siguientes tablas que visualizan expresiones numéricas de acuerdo a la asociación conjunta que las distinguen y así conocer la correspondencia que tienen las preguntas de las variables.

Tabla 3

Fui capaz de hacer bien mi trabajo y tengo confianza en los procesos de retroalimentación

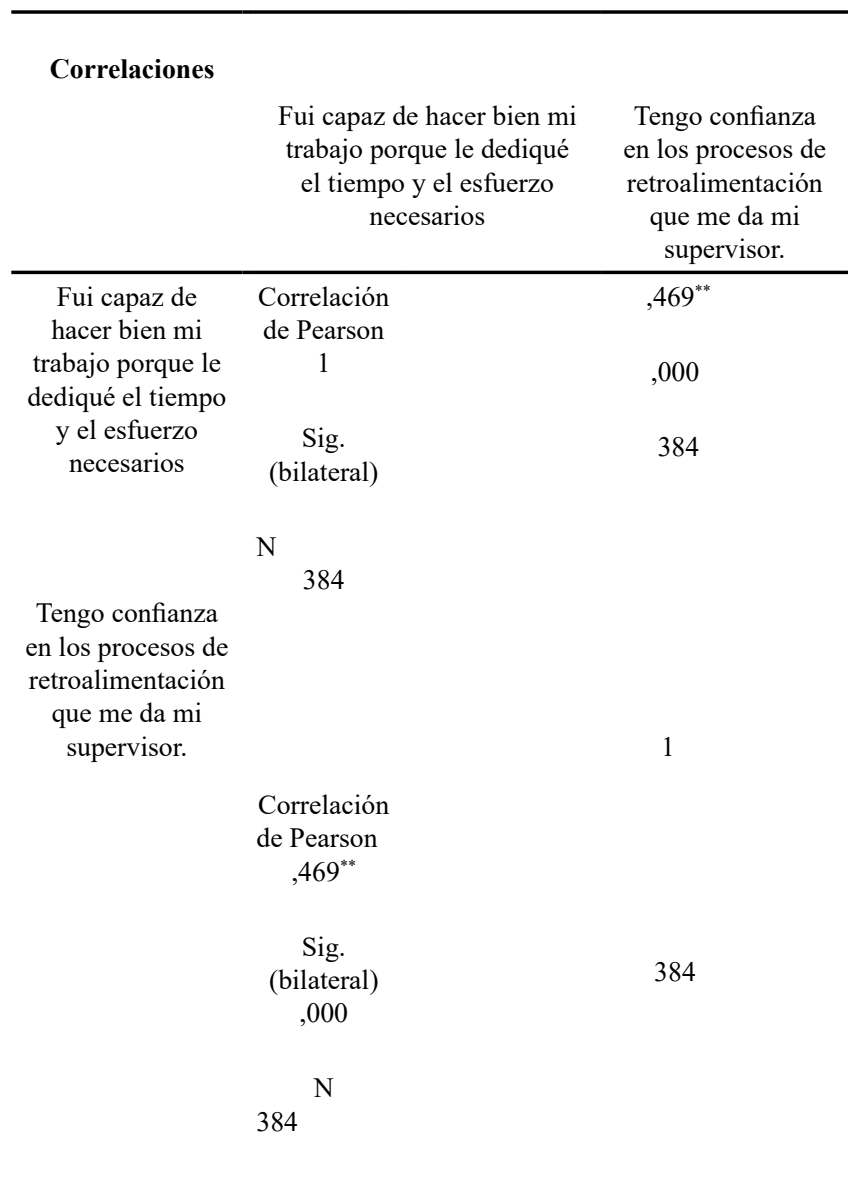

**. La correlación es significativa en el nivel 0,01 (bilateral).

Nota. Desarrollado a través del programa estadístico IBM SPSS con los datos obtenidos de la encuesta a los empleados de las Pymes.
La interpretación de los valores obtenidos por medio de la utilización de procedimientos estadísticos, acceden afirmar una relación positiva media entre las preguntas de la variable dependiente (Fui capaz de hacer bien mi trabajo porque le dediqué el tiempo y el esfuerzo necesarios) e independiente (Tengo confianza en los procesos de retroalimentación que me da mi supervisor) con un valor numérico de 0,469 ( ver Tabla 3), el cual presenta que el empleado busca cumplir con sus responsabilidades toda la jornada laboral y realizar acciones acorde a sus funciones, teniendo el respaldo del supervisor, ya que un $34,38 \%$ de los encuestados, manifestaron estar de acuerdo con la aportación de la información receptada.

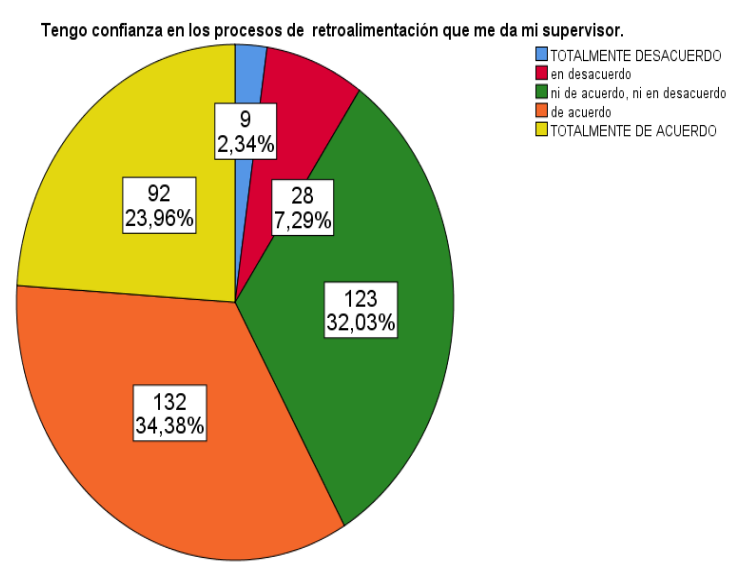

Figura 1. Tengo confianza en la retroalimentación que me da mi supervisor

Nota. Desarrollado a través del programa estadístico IBM SPSS con los datos obtenidos de la encuesta a los empleados de las Pymes. 


\section{Tabla 4}

Se me ocurrieron soluciones creativas frente a los nuevos problemas con recibo comentarios positivos de mi supervisor

\begin{tabular}{|c|c|c|c|}
\hline \multicolumn{4}{|c|}{ Correlaciones } \\
\hline & \multicolumn{2}{|c|}{$\begin{array}{c}\text { Se me ocurrieron } \\
\text { soluciones creativas } \\
\text { frente a los nuevos } \\
\text { problemas }\end{array}$} & $\begin{array}{c}\text { Recibo } \\
\text { comentarios } \\
\text { positivos de mi } \\
\text { supervisor. }\end{array}$ \\
\hline \multirow{5}{*}{$\begin{array}{c}\text { Se me ocurrieron } \\
\text { soluciones creativas } \\
\text { frente a los nuevos } \\
\text { problemas }\end{array}$} & \multicolumn{2}{|c|}{ Correlación de } & \\
\hline & \multicolumn{2}{|c|}{, $404^{* *}$} & \\
\hline & \multirow{2}{*}{\multicolumn{2}{|c|}{ Sig. (bilateral) }} & \\
\hline & & & \\
\hline & $\begin{array}{l}\mathrm{N} \\
384\end{array}$ & 384 & \\
\hline \multicolumn{4}{|l|}{$\begin{array}{l}\text { Recibo comentarios } \\
\text { positivos de mi } \\
\text { supervisor. }\end{array}$} \\
\hline \multicolumn{4}{|c|}{ Correlación de } \\
\hline \multicolumn{4}{|c|}{1} \\
\hline \multicolumn{4}{|c|}{$\begin{array}{c}\text { Sig. (bilateral) } \\
, 000\end{array}$} \\
\hline \multicolumn{4}{|c|}{$\mathbf{N}$} \\
\hline & 384 & 384 & \\
\hline
\end{tabular}

**. La correlación es significativa en el nivel 0,01 (bilateral).

Nota. Desarrollado a través del programa estadístico IBM SPSS con los datos obtenidos de la encuesta a los empleados de las Pymes.

La correlación entre: el argumento de si se me ocurrieron soluciones creativas frente a los nuevos problemas, ítem que se encuentra dentro de la variable dependiente (rendimiento); $\mathrm{y}$, recibir comentarios positivos de mi supervisor, perteneciente a la variable independiente (feedback) es positiva media con un valor de 0,404 (ver Tabla 4), esta relación se debe a que un $30,99 \%$ de los encuestados recibe apreciaciones acerca de su proactividad en los diferentes cambios económicos, sociales, políticos y culturales que se presentan.

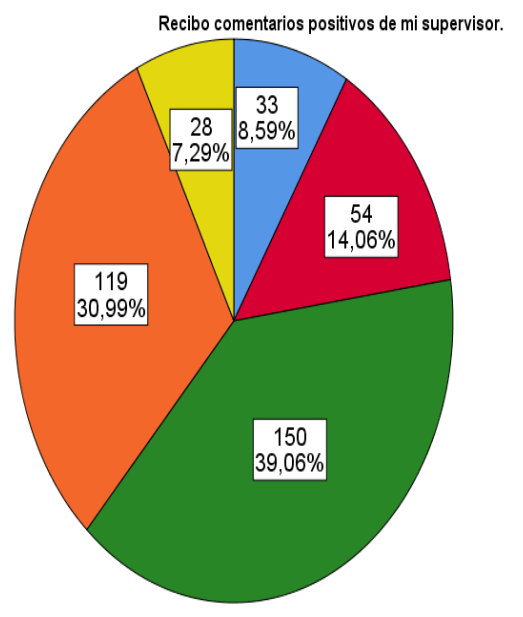

DTOTALMENTE DESACUERDO en desacuerdo Div de acuerdo, ni en desacuerdo TTOTALMENTE DE ACUERDO

Figura 2. Recibo comentarios positivos de mi supervisor

Nota. Desarrollado a través del programa estadístico IBM SPSS con los datos obtenidos de la encuesta a los empleados de las Pymes.

Tabla 5

Habilidades laborales actualizadas con mi supervisor es justo al evaluar mi desempeño laboral

\begin{tabular}{ccc}
\hline & Correlaciones & \\
& $\begin{array}{c}\text { Trabajé para mantener } \\
\text { mis habilidades } \\
\text { laborales actualizadas. }\end{array}$ & $\begin{array}{c}\text { Mi supervisor es } \\
\text { justo al evaluar mi } \\
\text { desempeño laboral. }\end{array}$ \\
\hline $\begin{array}{c}\text { Trabajé para } \\
\text { mantener mis } \\
\text { habilidades laborales } \\
\text { actualizadas. }\end{array}$ & $\begin{array}{c}\text { Correlación de Pearson } \\
1\end{array}$ &, $566^{* *}$ \\
& Sig. (bilateral) & \\
Mi supervisor es & $\mathrm{N}$ & 384 \\
justo al evaluar mi \\
desempeño laboral.
\end{tabular}

**. La correlación es significativa en el nivel 0,01 (bilateral).

Nota. Desarrollado a través del programa estadístico IBM SPSS con los datos obtenidos de 
la encuesta a los empleados de las Pymes.

La Tabla 5 evidenció una correlación positiva considerable con un valor de 0,566 entre las preguntas: trabajé para mantener mis habilidades laborales actualizadas; $\mathrm{y}$, mi supervisor es justo al evaluar mi desempeño laboral, donde los empleados buscan su autorrealización expandiendo sus conocimientos, habilidades y destrezas en capacitaciones, debido a esa flexibilidad el cumplimiento de actividades es mayor, por consecuente la evaluación debería ser realizada por el encargado de forma correcta, aunque solo un 26,04\% (ver Figura 3) de aquellos empleados que se preparan reciben la valoración justa.

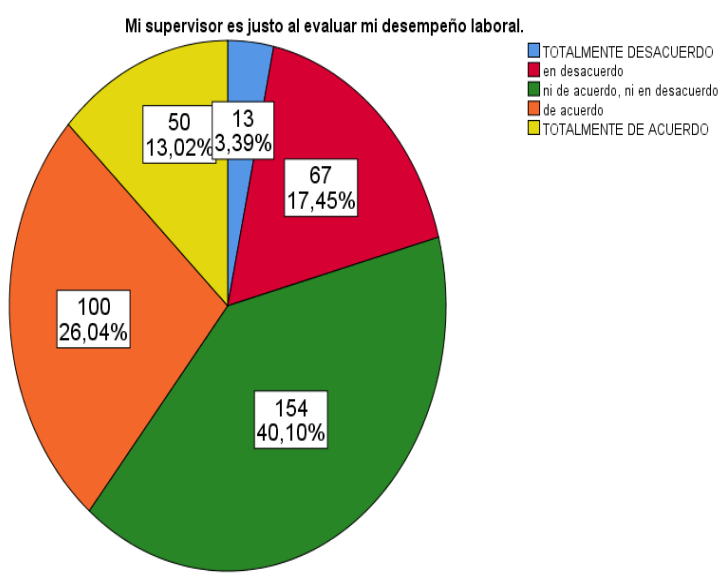

Figura 3. Mi supervisor es justo al evaluar mi desempeño laboral

Nota. Desarrollado a través del programa estadístico IBM SPSS con los datos obtenidos de la encuesta a los empleados de las Pymes.
Tabla 6

Participe activamente de las reuniones laborales con mi supervisor es justo al evaluar mi desempeño laboral

\begin{tabular}{|c|c|c|}
\hline \multicolumn{3}{|c|}{ Correlaciones } \\
\hline & $\begin{array}{c}\text { Participé activamente } \\
\text { de las reuniones } \\
\text { laborales. }\end{array}$ & $\begin{array}{c}\text { Mi } \\
\text { supervisor } \\
\text { es justo al } \\
\text { evaluar mi } \\
\text { desempeño } \\
\text { laboral. }\end{array}$ \\
\hline \multirow[t]{2}{*}{$\begin{array}{l}\text { Participé activamente de las } \\
\text { reuniones laborales. }\end{array}$} & $\begin{array}{l}\text { Correlación de Pearson } \\
1\end{array}$ &, $552^{* *}$ \\
\hline & Sig. (bilateral) &, 000 \\
\hline \multirow{5}{*}{$\begin{array}{l}\text { Mi supervisor es justo al } \\
\text { evaluar mi desempeño } \\
\text { laboral. }\end{array}$} & $\begin{array}{c}\mathrm{N} \\
384\end{array}$ & 384 \\
\hline & $\begin{array}{l}\text { Correlación de Pearson } \\
\qquad 552^{* *}\end{array}$ & \\
\hline & $\begin{array}{l}\text { Sig. (bilateral) } \\
, 000\end{array}$ & 1 \\
\hline & $\mathrm{N}$ & \\
\hline & 384 & 384 \\
\hline
\end{tabular}

Nota. Desarrollado a través del programa estadístico IBM SPSS con los datos obtenidos de la encuesta a los empleados de las Pymes.

Los empleados encuentran motivación al recibir por parte del supervisor estimaciones al momento de desarrollarse las valoraciones acerca de los procesos sistemáticos, así lo presenta la Tabla 6 con un valor de 0,552 lo cual significa que entre: participé activamente de las reuniones laborales; $y$, mi supervisor es justo al evaluar mi desempeño laboral hay una correlación positiva considerable ya que un $23,44 \%$ de los empleados casi siempre interfieren en las reuniones y un $26,04 \%$ están de acuerdo con la valoración por parte del encargado (ver Figura 3 ). 


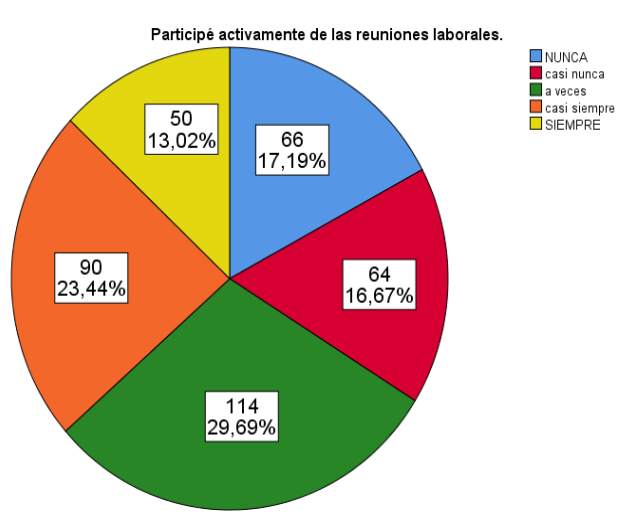

Figura 4. Participé activamente de las reuniones laborales.

Nota. Desarrollado a través del programa estadístico IBM SPSS con los datos obtenidos de la encuesta a los empleados de las Pymes

\section{Tabla 7}

Planifiqué mi trabajo de manera tal que pude hacerlo en tiempo y forma con tengo la confianza en los procesos de retroalimentación que me da mi supervisor

\begin{tabular}{|c|c|c|}
\hline \multicolumn{3}{|c|}{ Correlaciones } \\
\hline & $\begin{array}{c}\text { Planifiqué } \\
\text { mi trabajo } \\
\text { de manera } \\
\text { tal que } \\
\text { pude } \\
\text { hacerlo en } \\
\text { tiempo y } \\
\text { forma. }\end{array}$ & $\begin{array}{c}\text { Tengo } \\
\text { confianza en } \\
\text { los procesos de } \\
\text { retroalimentación } \\
\text { que me da mi } \\
\text { supervisor. }\end{array}$ \\
\hline \multirow{4}{*}{$\begin{array}{c}\text { Planifiqué mi } \\
\text { trabajo de manera } \\
\text { tal que pude } \\
\text { hacerlo en tiempo } \\
\text { y forma. }\end{array}$} & Correlación de Pearson &, $477^{* *}$ \\
\hline & Sig. (bilateral) &, 000 \\
\hline & 384 & 384 \\
\hline & $\begin{array}{l}\text { Correlación de Pearson } \\
\qquad 477^{* *}\end{array}$ & 1 \\
\hline \multirow{2}{*}{$\begin{array}{l}\text { Tengo confianza } \\
\text { en los procesos de } \\
\text { retroalimentación } \\
\text { que me da mi } \\
\text { supervisor. }\end{array}$} & $\begin{array}{l}\text { Sig. (bilateral) } \\
, 000\end{array}$ & \multirow[t]{2}{*}{384} \\
\hline & $\begin{array}{c}\mathrm{N} \\
384\end{array}$ & \\
\hline
\end{tabular}

**. La correlación es significativa en el nivel 0,01 (bilateral).

Nota. Desarrollado a través del programa estadístico IBM SPSS con los datos obtenidos de la encuesta a los empleados de las Pymes.

Planifiqué mi trabajo de manera tal que pude hacerlo en tiempo y forma, presenta el $42,71 \%$ de que casi siempre los empleados distribuyen su manera de trabajo, de igual manera el 34,38\% tienen confianza en los procesos que les da el supervisor (ver Figura 1), la Tabla 7 nos muestra un valor de 0,477 , la que arroja una correlación positiva media, en este caso se contempla que al tener una planificación se toma en cuenta los factores de tiempo y forma, abriéndose un espacio en que toda información que otorgue el supervisor será bien recibida para cumplir con los objetivos organizacionales.

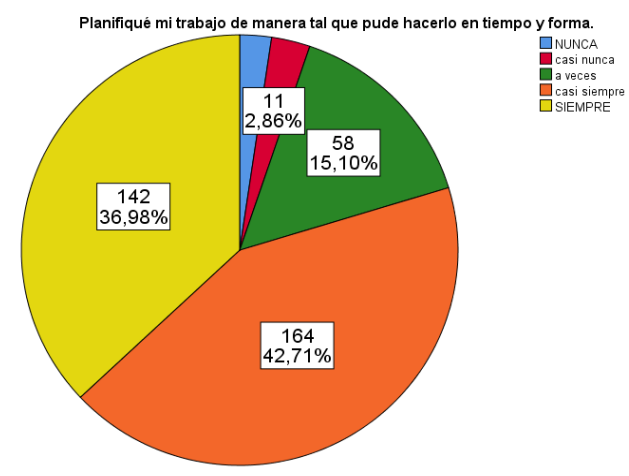

Figura 5. Planifiqué mi trabajo de manera tal que pude hacerlo en tiempo y forma

Nota. Desarrollado a través del programa estadístico IBM SPSS con los datos obtenidos de la encuesta a los empleados de las Pymes 
Tabla 8

Trabaje para mantener mis habilidades actualizadas con relación a tengo confianza en los procesos de retroalimentación

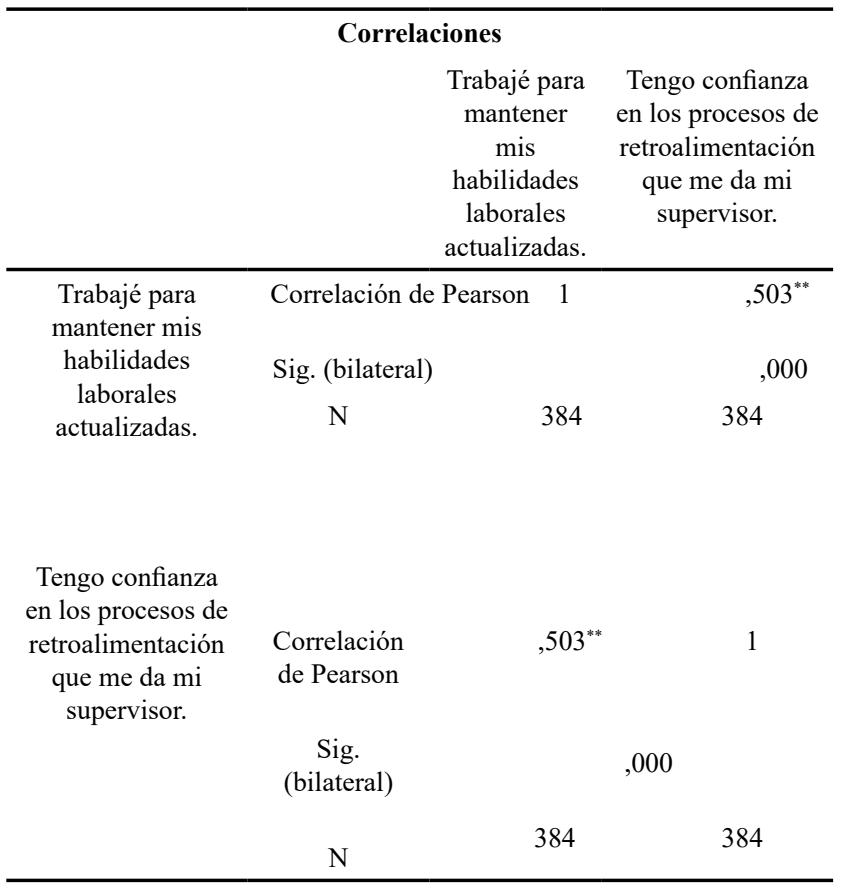

**. La correlación es significativa en el nivel 0,01 (bilateral).

Nota. Desarrollado a través del programa estadístico IBM SPSS con los datos obtenidos de la encuesta a los empleados de las Pymes.

Los empleados de las Pymes de comercio y de servicios en los últimos 3 meses han trabajado en obtener mayor conocimiento en el campo profesional para generar mayor competitividad, el cual cuenta con el soporte de confianza que les otorgan los encargados por cada actividad realizada, al demostrar mayor captación en las funciones, obteniendo un porcentaje según la Figura 1 de $23,70 \%$ que a veces trabajan para mantener sus habilidades actualizadas; $y, 34,38 \%$ tienen confianza en la información que reciben, en la Tabla 8 se muestra un valor de 0,503 en el rango de relación entre las dos preguntas de las diferentes variables de forma positiva media.

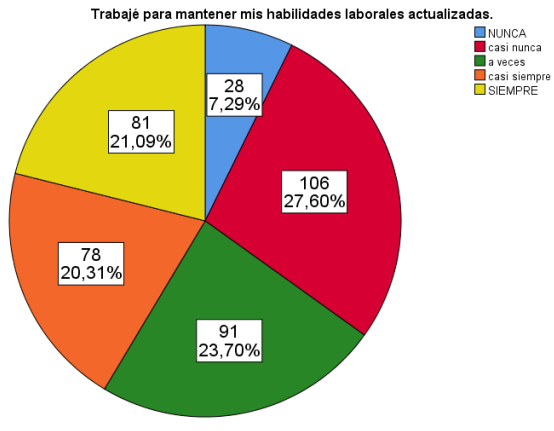

Figura 6. Trabajé para mantener mis habilidades laborales actualizadas

Nota. Desarrollado a través del programa estadístico IBM SPSS con los datos obtenidos de la encuesta a los empleados de las Pymes

Tabla 9

Participe activamente de las reuniones laborales con relación a recibo comentarios positivos de mi supervisor

\begin{tabular}{ccc}
\hline & Correlaciones & \\
& $\begin{array}{c}\text { Participé activamente } \\
\text { de las reuniones } \\
\text { laborales. }\end{array}$ & $\begin{array}{c}\text { Recibo comentarios } \\
\text { positivos de mi } \\
\text { supervisor. }\end{array}$ \\
\hline $\begin{array}{c}\text { Participé } \\
\text { activamente de } \\
\text { las reuniones } \\
\text { laborales. }\end{array}$ & Correlación de Pearson \\
Sig. (bilateral) &, $441^{* *}$ \\
N &, 000 \\
Recibo & Correlación de Pearson \\
comentarios & $441^{* *}$ & 384 \\
positivos de mi & Sig. (bilateral) & \\
supervisor. &, 000 & \\
& $\mathrm{~N}$ & \\
& 384 & \\
\end{tabular}

**. La correlación es significativa en el nivel 0,01 (bilateral).

Nota. Desarrollado a través del programa estadístico IBM SPSS con los datos obtenidos de la encuesta a los empleados de las Pymes.

La interacción del empleado en las reuniones, juntas o gestiones de la empresa da paso a que el supervisor otorgue comentarios positivos de aquella intervención la que se muestra una relación positiva media de 0,441 en la Tabla 9, por lo que entre mayor es la iniciativa de participar en determinados asuntos por parte del grupo de talento humano, estas tendrán 
opiniones acertadas de acuerdo a sus acciones, por lo que se demuestra en la Figura 4 un 23,44\% de participación en la reuniones y en la Figura 2 un valor $30,99 \%$ que están de acuerdo con las apreciaciones recibidas.

\section{Discusión}

La investigación se guio con las variables dependiente (rendimiento) e independiente (feedback), resolviendo las siguientes hipótesis H1: El feedback tiene impacto en el rendimiento de los empleados, Ho: El feedback no tiene impacto en el rendimiento de los empleados, tras haberse analizado cada una de las preguntas que se encontraban en dichas variables a través del método estadístico de correlación de Pearson en el programa IBM SPSS se obtuvieron los siguientes resultados: Tabla 3: 0,469, Tabla 4: 0.404, Tabla 5: 0.566, Tabla 6: 0.552, Tabla 7: 0.477, Tabla 8: 0.503; y, Tabla 9: 0.441, en base al análisis es oportuna la generalización de rangos con los intervalos de menor a mayor valor, en los que el estudio se centró siendo estos de 0,404 a 0,566, lo que presentó una correlación positiva media (ver Tabla 2); también, se evidenció que los empleados se encuentran de acuerdo con el feedback que reciben por parte de los supervisores.

Así que la relación que tienen las variables permite constatar las hipótesis declaradas, mediante la significancia bilateral de las tablas antes mencionadas, plasmándose un valor numérico de 0,000 , siendo este menor al margen de error de 0,05 en la muestra, por ese motivo se procede a aceptar la hipótesis alternativa (H1:El feedback tiene impacto en el rendimiento de los empleados), en razón de aquello se rechaza nula (Ho: El feedback no tiene impacto en el rendimiento de los empleados) dicho de otra manera no hay probabilidad de que la información que les brindan no tenga algún beneficio, por eso los empleados respondieron a través de las propiedades psicométricas que el feedback si tiene impacto en las actividades laborales como en el desarrollo de conocimientos, en consecuencia a esto su rendimiento es mayor al recibir este tipo de estrategia empresarial, visto que aporta de forma significativa al cumplimiento de los objetivos organizacionales.

Así que, aunque las empresas de comercio y servicio de la ciudad de Machala no realicen la retroalimentación de forma directa o constante, se consto que, si incide en los empleados que haya una comunicación bilateral, por lo cual se evidencia la necesidad de realizar otras investigaciones para conocer otros factores que predominan en las Pymes para que exista un rendimiento eficaz y eficiente por parte del grupo de talento humano.

\section{Conclusiones}

El estudio evidencia que la orientación lectora ha sido posible en virtud del marco teórico y contextualización, los cuales fueron desarrollados en base a artículos académicos y libros, exponiendo la importancia del feedback en las gestiones del talento humano dentro de la organización.

Los datos estadísticos fueron adquiridos mediante la encuesta, que fue ajustada de los instrumentos de Steelman, Levy, y Snell (2004) feedback environment; y; Gabini y Salessi (2016) escala de rendimiento laboral individual con lo que se recopiló la información desde la percepción de los empleados.

Los resultados se obtuvieron con el apoyo del programa estadístico IBM SPSS por el cual se determinó la relación existente entre el feedback y rendimiento por medio del método correlación de Pearson, teniendo una medida positiva media entre las preguntas que se encuentran estipuladas dentro de las variables.

Por lo tanto, el estudio ha sido fundamental ya que a través de la medición del rendimiento laboral se estudió la estrategia feedback y determinó el impacto que tiene en las Pymes de comercio y servicios de la ciudad de Machala.

\section{Referencias Bibliográficas}

Aguilar, S. (2005). Fórmulas para el cálculo de la muestra en investigaciones de salud. Salud en Tabasco, 11(1-2), 333-338. Obtenido de https://www.redalyc.org/ 
pdf/487/48711206.pdf

Amo, A. (2018). Habilidades de comunicación (Vol. 1.0). España: Editorial Elearning, S.L. Obtenido de https://books.google. com.ec/books?id=RG12DwAAQBAJ\&pg $=$ PA57\&dq $=$ Feedback + positivo: + es + reco nocer $+\mathrm{a}+\mathrm{la}+$ otra + persona + una + determina $\mathrm{da}+$ conducta, $+\mathrm{lo}+$ que + har $\% \mathrm{C} 3 \% \mathrm{~A} 1+$ que + su+entusiasmo $+\mathrm{y}+$ motivaci $\% \mathrm{C} 3 \% \mathrm{~B} 3 \mathrm{n}+$ aumenten. $+\mathrm{Se}+$ puede + hacer $+\mathrm{en}+\mathrm{p} \% \mathrm{C} 3 \%$ BAblico + o+en + privado, + si + bien + en

Bravo, L., Valenzuela, A., Ramos, P., \& Tejada, A. (2019). Perspectiva teórica del diagnóstico organizacional. Revista Venezolana de Gerencia, 24(88), 1-11. doi: ISSN: 13159984

Brito, A., Narváez, C., Erazo, J., \& Torres, M. (2020). Impacto de los impuestos directos e indirectos en las Pyme de la ciudad de Cuenca período 2016-2018. 593 Digital Publisher, 5(4-1), 119-136. doi:doi. org/10.33386/593dp.2020.4-1.301

Caldas, M., Navarro, A., \& Hidalgo, M. (2016). Formación y orientación laboral. s/c: Editex. Obtenido de https://books.google. com.ec/books?id=NglCDAAAQBAJ\&pg $=$ PA228\&dq=permite + informar + al + recept or + de + la + calidad + de + su + trabajo, + de + ma nera + que + se + mantengan + los + comportam ientos + considerados + como + positivos $+y+$ $\mathrm{se}+$ intenten + mejorar + aquellos + que + no $+s$ e+est $\%$ C3\%A $1 n+$ realizan

Casares, E. (abril-mayo de 2007). La Comunicación en la Organización; la Retroalimentación como Fuente de Satisfacción. Razón y Palabra, 1-8.

De Alba, C. (2015). Seguimiento del proceso de inserción sociolaboral de personas con discapacidad. España: Ediciones Paraninfo. Obtenido de https://books. google.com.ec/books?id=CdI9CwAAQB AJ\&pg=PA20\&dq=rendimiento+laboral \& $\mathrm{hl}=\mathrm{es} \& \mathrm{sa}=\mathrm{X} \& \mathrm{ved}=2 \mathrm{ahUKEwixi5PrlIfsA}$ hUOr1kKHcO5DkUQ6AEwAXoECAM
$\mathrm{QAg \# v}=$ onepage \&q\&f=false

Gabini, S., \& Salessi, S. (2016). Validación de la Escala de Rendimiento Laboral Individual en Tra- bajadores Argentinos. Evaluar, 16(1), 10-26. Obtenido de https://www. researchgate.net/publication/334078598 Validacion_de_la_escala_de rendimiento_laboral_individual_en_ trabajadores_argentinos

Gabriel, J. (2017). Cómo se genera una investigación científica que luego sea motivo de publicación. Journal of the Selva Andina Research Society, 8(2), 145146. Obtenido de https://www.redalyc.org/ pdf/3613/361353711008.pdf

Garbanzo, G. (2016). Desarrollo organizacional y los procesos de cambio en las instituciones educativas, un reto de la gestión de la educación. Educación, 40(1), 67-87. Obtenido de https://www.redalyc.org/ pdf/440/44043204005.pdf

García, J., Galarza, S., \& Altamirano, A. (Agosto de 2017). Importancia de la administración eficiente del capital de trabajo en las Pymes. Revista Ciencia UNEMI, 10(23), 30 - 39. doi:ISSN 2528-7737

García, S. (2017). La inteligencia de valores (Vol. 1.0). Alicante, España: Club Universitario. Obtenido de https://books.google.com.ec/ books? id=mrBdDwAAQBAJ\&pg=PA29 $8 \& \mathrm{dq}=$ caracteristicas + del + feedback + lab oral\&hl $=$ es \&sa $=$ X\&ved $=0$ ahUKEwjwrb 6e2-XmAhXNs1kKHQO9CBUQ6AEIO $\mathrm{DAC} \# \mathrm{v}=$ onepage $\& \mathrm{q}=$ caracteristicas $\% 20$ del $\% 20$ feedback $\% 20$ laboral $\& \mathrm{f}=$ false

Giraudier, M. (2018). Cómo Gestionar el Clima Laboral. Integra.

Hernández, E. (2016). Manual. Valoración, seguimiento, y difusión de acciones de mediación. Madrid: EDITORIAL CEP. Obtenido de https://books.google.com. ec/books?id=-cU-DwAAQBAJ\&pg $=$ P $\mathrm{A} 43 \& \mathrm{dq}=$ mediciones $+\mathrm{del}+$ rendimiento + objetivas $+\mathrm{y}+$ subjetivas $\& \mathrm{hl}=\mathrm{es} \& \mathrm{sa}=\mathrm{X}$ 
\&ved=2ahUKEwjNxbimop7sAhVCw VkKHQufAAkQ6AEwBHoECAUQAg${ }_{\mathrm{H}} \mathrm{v}=$ onepage\&q\& $\mathrm{f}=$ false

Ibarra, M., González, L., \& Demuner, M. (2017). Competitividad empresarial de las pequeñas y medianas empresas manufactureras de Baja California. Estudios fronterizos, 18(35), 107-130. doi:https://doi.org/10.21670/ref.2017.35. a06

Jiménez, F. (enero-abril de 2015). Uso del Feedback como Estrategia de evaluación: Aportes desde un Enfoque. Revista Electrónica "Actualidades Investigativas en Educación”, 15(1), 1-24. Obtenido de https://www.redalyc.org/ pdf/447/44733027035.pdf

Koopmans, L., Bernaards, C., Hildebrandt, V., Buuren, S., Beek, A., \& Vet, H. (2012). Development of an Individual Work Performance Questionnaire. International Journal of Productivity and Performance Management, 6-28. Obtenido de https://www.researchgate.net/ publication/233944832_Development of_an_Individual_Work_Performance_ Questionnaire

Mondragón, M. (2014). USO DE LA CORRELACIÓNDESPEARMANENUN ESTUDIO. Articulos de Reflexion, 8(1), 98104. Obtenido de https://dialnet.unirioja. es/servlet/articulo?codigo $=5156978$

Mora, E., Vera, M., \& Melgarejo, Z. (2015). Planificación estratégica y niveles de competitividad de las Mipymes del sector comercio en Bogotá. Estudios Gerenciales, 31(134), 79-87. Obtenido de https://www. redalyc.org/pdf/212/21233043009.pdf

Muñoz, M., Cabrita, M., \& Ribeiro, M. (2015). Técnicas de gestión empresarial en la globalización. Revista de Ciencias Sociales, 346-357. Obtenido de https:// www.redalyc.org/pdf/280/28042299006. pdf
Rivero, Y. (2019). Evaluación del desempeño: tendencias actuales. Revista Archivo Médico de Camagüey, 23(2), 159164. Obtenido de http://scielo.sld. $\mathrm{cu} /$ scielo.php? script $=$ sci_arttext\&pid $=\mathrm{S} 1025-02552019000200159$

Sánchez, M., Sánchez, P., Cruz, M., \& Sánchez, F. (2014). Caracteristicas Organizacionales de la Satisfacciòn Laboral en España. Revista de Administração de Empresas, 537-547. Obtenido de https://www. redalyc.org/articulo.oa?id=155131802006

Steelman, L. A., Levy, P. E., \& Snell, A. F. (2004). The Feedback Environment Scale: Construct Definition, Measurement, and Validation. Educational and Psychological Measurement, 64(1), 165-184. Obtenido de https://www.researchgate.net/ publication/247728554_The_Feedback Environment_Scale_Cönstruct Definition_Measurement_and_Validation

Urcola, J. (2015). Manual práctico de comunicación empresarial. Madrid: ESIC. Obtenido de https://books.google.com.ec/ books?id=LayMCgAAQBAJ\&pg=PT155 $\& \mathrm{dq}=$ feedback + empresarial $\& \mathrm{hl}=\mathrm{es} \& \mathrm{sa}=$ X\&ved $=0$ ahUKEwiEhIuBtMrmAhVCVs 0KHdhmA1wQ6AEIJzAA\#v=onepage\&q $=$ feedback $\% 20$ empresarial $\& \mathrm{f}=$ false

Urdaneta, A., González, A., Luciani, L., \& Borgucci, E. (2019). Valor Agregado y Nivel Competitividad de las pymes en el Cantón Machala, Ecuador. Visionario Digital, 3(2), 245-265. doi:https://doi. org/10.33262/visionariodigital.v3i2.422

Zapata Guerrero, E. E. (2004). Las PyMES y su problemática empresarial. Análisis de casos. Revista Escuela de Administración de Negocios, 119-135. Obtenido de https:// www.redalyc.org/pdf/206/20605209.pdf

Zuluaga, M. (2017). El estudiante y la retroalimentación. Papel en la educación médica. Acta Médica Colombiana, 42(3), 193-194. 